\title{
Rendimento de forragem e morfogênese de Brachiaria brizantha cv. Marandu sob níveis de desfolhação nos cerrados de Roraima
}

$\underline{\text { Newton de Lucena Costa }}^{1^{*}}$; Claudio Ramalho Townsend ${ }^{2 * *}$; Fabíola Helena dos Santos Fogaça $^{3}$; João Avelar Magalhães ${ }^{4}$; Francisco José de Seixas Santos ${ }^{5}$; Braz Henrique Nunes Rodrigues

${ }^{I}$ Eng. Agr., D.Sc., Pesquisador da Embrapa Roraima, Boa Vista, RR.

${ }^{2}$ Zootecnista, D.Sc., Pesquisador da Embrapa Clima Temperado, Pelotas, RS.

${ }^{3}$ Zootecnista, D.Sc., Pesquisador da Embrapa Meio-Norte, Parnaíba, PI.

${ }^{4}$ Méd. Vet., D.Sc., Pesquisador da Embrapa Meio-Norte, Parnaíba, PI.

${ }^{5}$ Eng. Agr., D.Sc., Pesquisador da Embrapa Meio-Norte, Parnaíba, PI.

${ }^{6}$ Eng.Agrícola, D.Sc., Pesquisador da Embrapa Meio-Norte, Parnaíba, PI.

*Autor para correspondência, E-mail: newtonlucena@yahoo.com.br

**in memorian

RESUMO. O efeito de níveis de desfolhação $(10,15,20,25$ e $30 \mathrm{~cm}$ acima do solo) sobre a produção de forragem e características morfogênicas e estruturais de Brachiaria brizantha cv. Marandu foi avaliado nos cerrados de Roraima. Os efeitos dos níveis de desfolhação sobre a produção de matéria seca, número de folhas/perfilho, tamanho médio de folhas, índice de área foliar e taxas de aparecimento e expansão de folhas foram quadráticos e os máximos valores registrados com cortes a 23,$1 ; 18,6 ; 26,3 ; 24,1 ; 14,8$ e $21,3 \mathrm{~cm}$ acima do solo, respectivamente. A densidade populacional de perfilhos foi inversamente proporcional ao nível de desfolhação, ocorrendo o inverso quanto à taxa de senescência foliar. A eliminação de meristemas apicais foi incrementada com o aumento do nível de desfolhação. $\mathrm{O}$ vigor de rebrota foi direta e negativamente correlacionado com o nível de desfolhação.

Palavras chave: folhas, matéria seca, perfilhamento, senescência

\section{Forage yield and morphogenesis of Brachiaria brizantha cv. Marandu under defoliation levels in Roraima's savannas}

\begin{abstract}
The effect of defoliation levels (10, 15, 20, 25 and $30 \mathrm{~cm}$ above soil level) on dry matter (DM) yield and morphogenetic and structural characteristics of Brachiaria brizantha cv. Marandu was evaluated under field conditions in Roraima's savannas. The effects of defoliation levels on the DM yields, number of leaves/plant, medium blade length, leaf area index, leaf appearance and elongation rates were quadratics and maximum values recorded with cutting at $23.1 ; 18.6 ; 26.3 ; 24.1 ; 14.8$ and $21.3 \mathrm{~cm}$ above soil level, respectively. The population tiller density was inversely proportional to the level of defoliation, while the opposite occurred for to leaf senescence rate. Apical meristem removing percentage was higher with increasing defoliation level. Aftermath regrowth showed close negative correlation with defoliation level.
\end{abstract}

Keywords: dry matter, leaves, tillering, senescence

\section{Introdução}

Em Roraima, a pecuária é uma das atividades em franca expansão e as pastagens cultivadas representam o principal recurso forrageiro para a alimentação dos rebanhos. A utilização do pastejo contínuo ou períodos mínimos de descanso e altas intensidades de desfolhação são fatores que contribuem para baixa disponibilidade e qualidade da forragem, com reflexos negativos nos índices de desempenho zootécnico dos animais (Costa et al., 2009). A produtividade e a perenidade das pastagens decorrem, entre outros 
fatores, da capacidade de reconstituição e manutenção da área foliar após a desfolhação, a qual afeta a estrutura do dossel, determinando sua velocidade de crescimento, acúmulo de forragem, composição química e persistência (Zanini et al., 2012). A intensidade de desfolhação indica a proporção do tecido vegetal removido pelo animal em relação ao disponibilizado para pastejo, impactando a área foliar fotossinteticamente ativa remanescente, a remobilização de reservas orgânicas e a remoção de meristemas apicais (Lemaire et al., 2011).

A morfogênese de uma gramínea durante seu crescimento vegetativo é caracterizada por três fatores: a taxa de aparecimento, a taxa de expansão e a longevidade das folhas. A taxa de aparecimento e a duração e vida das folhas condicionam o número de folhas vivas/perfilho, as quais são determinadas geneticamente e podem ser afetadas pelos fatores ambientais e práticas de manejo adotadas (Santos et al., 2012). O número de folhas vivas por perfilho, constante para cada espécie, constitui critério objetivo na definição dos sistemas de pastejo a serem impostos no manejo das forrageiras. Desta forma, estudos de dinâmica do crescimento de folhas e perfilhos de gramíneas forrageiras perenes são importantes para a definição de estratégias de manejo específicas e sustentáveis (Sousa et al., 2011).

Neste trabalho avaliou-se o efeito de níveis de desfolhação sobre a produção de forragem e características morfogênicas e estruturais de pastagens de Brachiaria brizantha cv. Marandu nos cerrados de Roraima.

\section{Material e Métodos}

O ensaio foi conduzido no Campo Experimental da Embrapa Roraima, localizado em Boa Vista, durante o período de maio a setembro de 2013. O solo da área experimental é um Latossolo Amarelo, textura média, com as seguintes características químicas, na profundidade de $0-20 \mathrm{~cm}: \mathrm{pH}_{\mathrm{H}_{2} \mathrm{O}}=4,9 ; \mathrm{P}=2,1$ $\mathrm{mg} / \mathrm{kg} ; \mathrm{Ca}+\mathrm{Mg}=1,15 \mathrm{cmol}_{\mathrm{c}} \cdot \mathrm{dm}^{-3} ; \mathrm{K}=0,019$ $\mathrm{cmol}_{\mathrm{c}} \cdot \mathrm{dm}^{-3}$ e $\mathrm{Al}=0,59 \mathrm{cmol}_{\mathrm{c}} \mathrm{dm}^{-3}$.

O delineamento experimental foi inteiramente ao acaso, com três repetições. Os tratamentos consistiram de cinco níveis de desfolhação (10, $15,20,25$ e $30 \mathrm{~cm}$ acima do solo). O tamanho das parcelas foi de $2,0 \mathrm{~m} \times 2,0 \mathrm{~m}$, sendo a área útil de $1,0 \mathrm{~m}^{2}$. A adubação de estabelecimento constou da aplicação de $50 \mathrm{~kg}$ de $\mathrm{P}_{2} \mathrm{O}_{5}$ ha $^{-1}$ e 60 $\mathrm{kg}$ de $\mathrm{K}_{2} \mathrm{O}$ ha $^{-1}$, respectivamente sob a forma de superfosfato triplo e cloreto de potássio. Durante o período experimental foram realizados três cortes a intervalos de 35 dias.

Os parâmetros avaliados foram rendimento de matéria seca (MS), densidade populacional de perfilhos (DPP), número de folhas/perfilho (NFP), taxa de aparecimento de folhas (TAF), taxa de expansão foliar (TEF), taxa de senescência foliar (TSF), tamanho médio de folhas (TMF) e índice de área foliar (IAF). A TEF e TAF foram obtidas dividindo-se $\mathrm{o}$ comprimento acumulado de folhas e o número total de folhas no perfilho, respectivamente, pelo período de rebrota. O TMF foi determinado pela divisão do alongamento foliar total do perfilho pelo seu número de folhas.

Para o cálculo da área foliar, em cada idade de rebrota foram coletadas amostras de folhas verdes completamente expandidas, procurando-se obter uma área entre 200 e $300 \mathrm{~cm}^{2}$, sendo estimada com planímetro ótico eletrônico (LiCor, modelo LI-3100C). Posteriormente, as amostras foram levadas à estufa com ar forçado a $65^{\circ} \mathrm{C}$ até atingirem peso constante, obtendo-se a MS foliar. A área foliar específica (AFE) foi determinada através da relação entre área de folhas verdes e sua MS ( $\mathrm{m}^{2} / \mathrm{g}$ MS foliar). O IAF foi determinado a partir do produto entre a MS total das folhas verdes $\left(\mathrm{g}\right.$ de $\mathrm{MS} / \mathrm{m}^{2}$ ) pela AFE $\left(\mathrm{m}^{2} / \mathrm{g}\right.$ de MS foliar). A TSF foi obtida dividindose o comprimento da folha que se apresentava de coloração amarelada ou necrosada pelo período de rebrota. A sobrevivência dos meristemas apicais foi estimada relacionando-se com o número total de perfilhos aqueles que se apresentavam com folhas novas truncadas, sete dias após o corte das plantas. O vigor de rebrota foi avaliado através da produção de MS aos 21 dias após o corte à idade do primeiro corte.

\section{Resultados e Discussão}

Os rendimentos de MS foram afetados $(\mathrm{P}<0,05)$ pelos níveis de desfolhação, sendo a relação quadrática $(\mathrm{Y}=781,3+212,94 \mathrm{X}$ $5,5864 X^{2} ; R^{2}=0,92$ ) e o máximo valor estimado com cortes a $23,1 \mathrm{~cm}$ acima do solo (Tabela 1 ). $\mathrm{O}$ primeiro efeito imediato da desfolhação sobre a planta é a diminuição de sua área foliar e, consequentemente, da capacidade em interceptar luz e a redução global da fotossíntese, as quais são afetadas pela proporção de tecido removido; o grau de desfolha das plantas vizinhas e a 
capacidade fotossintética do tecido foliar remanescente após a desfolhação (Costa, 2004, Lemaire et al., 2011, Sousa et al., 2011). Após a desfolhação ocorre rápido declínio na quantidade de carboidratos solúveis nas raízes, como consequência do decréscimo na taxa fotossintética da planta como um todo e alocação preferencial de carbono para as partes áreas da planta com a finalidade de restaurar sua área foliar (Santos et al., 2011). A redução no nível de desfolhação assegura a retenção de maior área foliar fotossinteticamente ativa e maior remobilização de nutrientes, resultando em maior velocidade de recuperação e menor intervalo entre pastejos (Nabinger \& Carvalho, 2009, Sousa et al., 2011). Costa et al. (2009) estimaram maiores rendimentos de MS para pastagens de Brachiaria brizantha cv. Xaraés mantidas sob resíduo de $30 \mathrm{~cm}\left(6.319 \mathrm{~kg} \mathrm{ha}^{-1}\right)$, comparativamente a $15 \mathrm{~cm}\left(2.991 \mathrm{~kg} \mathrm{ha}^{-1}\right)$. Para pastagens de Panicum maximum cv. Tanzânia, Canto et al. (2008) reportaram acréscimos lineares na disponibilidade de forragem com a redução da intensidade de desfolhação (2.810; $3.155 ; 3.678$ e $4.110 \mathrm{~kg} / \mathrm{MS} / \mathrm{ha}^{-1}$, respectivamente para 20, 40, 60 e $80 \mathrm{~cm}$ acima do solo).
A remoção de meristemas apicais foi inversamente proporcional ao nível de desfolhação ( $\left.\mathrm{Y}=86,62-1,58 \mathrm{X} ; \mathrm{R}^{2}=0,97\right)$. Costa et al. (2009) reportaram maior remoção de meristemas apicais para $P$. maximum cv. Centenário com cortes a $20 \mathrm{~cm}$ acima do solo $(79,3 \%)$, comparativamente a $30(50,7 \%)$ ou 40 $\mathrm{cm}(36,1 \%)$, a qual foi negativamente correlacionada com a produção de forragem. $\mathrm{O}$ vigor de rebrota foi afetado pelos níveis de desfolhação $(\mathrm{P}<0,05)$, sendo a relação quadrática e descrita pela equação $\mathrm{Y}=260,4+54,16 \mathrm{X}$ $1,241 \mathrm{X}^{2}\left(\mathrm{R}^{2}=0,86\right)$ e o máximo valor estimado aos $21,8 \mathrm{~cm}$. Costa et al. (2009) constataram que o vigor de rebrota de Andropogon gayanus cv. Planaltina foi diretamente proporcional ao nível de desfolhação (671; 921 e 1.132 kg de MS/21 dias, respectivamente para cortes a 10, 20 e 30 $\mathrm{cm}$ acima do solo). A remoção de meristemas apicais retarda a reconstituição foliar das gramíneas a qual terá origem a partir do desenvolvimento de gemas axilares ou basilares, as quais apresentam menor velocidade de crescimento (Lemaire et al., 2011).

Tabela 1. Rendimento de matéria seca (MS - $\mathrm{kg} \mathrm{ha}^{-1}$ ), remoção de meristemas apicais (RMA - \%), densidade populacional de perfilhos (DPP - $\mathrm{m}^{-2}$ ), número de folhas/perfilho (NFP), tamanho médio de folhas (TMF - $\mathrm{cm}$ ), índice de área foliar (IAF $-\mathrm{m}^{2} / \mathrm{m}^{2}$ ), taxa de aparecimento de folhas (TAF - folhas/perfilho.dia), taxa de expansão foliar (TEF - cm/perfilho.dia) e taxa de senescência foliar (TSF - cm/perfilho.dia) de Brachiaria brizantha cv. Marandu, em função do nível de desfolhação.

\begin{tabular}{cccccccccc}
\hline $\begin{array}{c}\text { Desfolhação } \\
(\mathrm{cm})\end{array}$ & MS $^{1}$ & RMA & DPP & NFP & TMF & IAF & TAF & TEF & TSF \\
\hline 10 & $2.357 \mathrm{~d}$ & $71,4 \mathrm{a}$ & $802 \mathrm{a}$ & $5,19 \mathrm{~b}$ & $15,2 \mathrm{~d}$ & $2,51 \mathrm{c}$ & $0,148 \mathrm{~d}$ & $2,25 \mathrm{c}$ & $0,121 \mathrm{~d}$ \\
15 & $2.788 \mathrm{c}$ & $64,3 \mathrm{~b}$ & $754 \mathrm{~b}$ & $5,33 \mathrm{~b}$ & $16,8 \mathrm{c}$ & $2,98 \mathrm{~b}$ & $0,152 \mathrm{~b}$ & $2,56 \mathrm{~b}$ & $0,139 \mathrm{c}$ \\
20 & $3.411 \mathrm{a}$ & $51,2 \mathrm{c}$ & $722 \mathrm{~b}$ & $5,81 \mathrm{a}$ & $18,2 \mathrm{~b}$ & $3,57 \mathrm{a}$ & $0,166 \mathrm{a}$ & $3,02 \mathrm{a}$ & $0,147 \mathrm{bc}$ \\
25 & $3.519 \mathrm{a}$ & $47,4 \mathrm{c}$ & $681 \mathrm{c}$ & $5,15 \mathrm{~b}$ & $19,6 \mathrm{a}$ & $3,78 \mathrm{a}$ & $0,147 \mathrm{~b}$ & $2,88 \mathrm{a}$ & $0,159 \mathrm{~b}$ \\
30 & $3.027 \mathrm{~b}$ & $40,2 \mathrm{~d}$ & $633 \mathrm{~d}$ & $4,88 \mathrm{c}$ & $17,7 \mathrm{~b}$ & $3,62 \mathrm{a}$ & $0,139 \mathrm{c}$ & $2,47 \mathrm{~b}$ & $0,177 \mathrm{a}$ \\
\hline
\end{tabular}

Médias seguidas de mesma letra não diferem entre si $(\mathrm{P}>0,05)$ pelo teste de Tukey. 1 - Médias de três cortes.

Para o NFP, IAF e TMF as relações foram ajustadas ao modelo quadrático de regressão e definidas, respectivamente, pelas equações: $\mathrm{Y}=$ $3,62+0,208 \mathrm{X}-0,0056 \mathrm{X}^{2}\left(\mathrm{R}^{2}=0,89\right), \mathrm{Y}=$ $0,444+0,2478 X-0,0047 X^{2}\left(R^{2}=0,97\right)$ e $Y=$ $7,48+0,9433 X-0,0196 X^{2}\left(R^{2}=0,90\right)$, sendo os máximos valores obtidos com desfolhações a 18,6; 26,3 e 24,1 cm acima do solo. Os valores registrados, neste trabalho, para o NFP, TMF e IAF foram superiores aos reportados por Sousa et al. (2011) para pastagens de Brachiaria brizantha cv. Xaraés mantidas sob resíduo de $20 \mathrm{~cm}$, que estimaram 5,11 folhas/perfilho; $11,2 \mathrm{~cm} /$ folha e IAF de 2,48. O TMF é uma característica plástica e responsiva à intensidade de desfolhação e considerada uma estratégia morfológica de escape das plantas ao pastejo. Em geral, os menores valores para o TMF foram constatados com maiores intensidades de desfolhação, provavelmente em decorrência da redução da fase de multiplicação celular e da distância que a lâmina deveria percorrer até emergir do 
pseudocolmo (Lemaire et al., 2011). A DPP foi inversamente proporcional ao nível de desfolhação, sendo a relação linear e descrita pela equação: $\mathrm{Y}=882,8-8,22 \mathrm{X}\left(\mathrm{r}^{2}=0,98\right)$. $\mathrm{O}$ potencial de perfilhamento da gramínea depende da velocidade de emissão de folhas, as quais produzirão gemas aptas a originar novos perfilhos, dependendo das condições ambientais e práticas de manejo adotadas (Costa, 2004, Nabinger \& Carvalho, 2009, Lemaire et al., 2011).

As relações entre níveis de desfolhação, TAF e TEF foram ajustadas ao modelo quadrático de regressão e descritas, respectivamente, pelas equações: $\mathrm{Y}=0,1038+0,0059 \mathrm{X}-0,0002 \mathrm{X}^{2}\left(\mathrm{R}^{2}\right.$ $=0,87)$ e $Y=0,3078+0,2467 X-0,0058 X^{2}\left(R^{2}\right.$ $=0,91)$ e os máximos valores estimados com cortes a 14,8 e $21,3 \mathrm{~cm}$ acima do solo (Tabela 1 ). A TAF é a característica morfogênica que merece maior destaque, pois afeta diretamente o tamanho da folha, a densidade populacional de perfilhos e o número de folhas/perfilho (Nabinger \& Carvalho, 2009, Difante et al., 2011). As TAF e TEF apresentam correlação negativa, indicando que quanto maior o surgimento de folhas, menor será o tempo disponível para a sua expansão (Lemaire et al., 2011).

A relação entre desfolhação e TSF foi linear: $\mathrm{Y}=0,0958+0,0026 \mathrm{X}\left(\mathrm{r}^{2}=0,98\right)$. Costa et al. (2009) constataram maior TSF para pastagens de Brachiaria decumbens sob resíduos de $30 \mathrm{~cm}$ $(0,198 \mathrm{~cm} /$ perfilho.dia), comparativamente a 20 $\mathrm{cm}(0,161 \mathrm{~cm} /$ perfilho.dia $)$ ou $10 \mathrm{~cm}(0,138$ $\mathrm{cm} /$ perfilho.dia). A senescência apesar do efeito negativo sobre a qualidade da forragem representa um importante processo fisiológico no fluxo de tecidos da gramínea, pois em torno de 35; 68; 86 e 42\% do nitrogênio, fósforo, potássio e magnésio, respectivamente, podem ser reciclados das folhas senescentes e utilizados para a produção de novos tecidos foliares (Sarmiento et al., 2006, Costa et al., 2013).

\section{Conclusões}

A desfolhação afeta a produção de matéria seca e as características morfogênicas e estruturais da gramínea. A eliminação de meristemas apicais foi diretamente proporcional ao nível de desfolhação, ocorrendo o oposto quanto ao vigor de rebrota. A manutenção de pastagens de $B$. brizantha cv. Marandu sob resíduos entre 20 e $25 \mathrm{~cm}$ asseguram maior produtividade e eficiência de utilização da forragem, maior renovação de tecidos e estrutura do dossel mais favorável ao pastejo.

\section{Referências Bibliográficas}

Canto, M. W., Jobim, C. C., Gasparino, E. \& Hoeschl, A. R. (2008). Características do pasto e acúmulo de forragem em capimtanzânia submetido a alturas de manejo do pasto. Pesquisa Agropecuária Brasileira, 43, 429-435.

Costa, N. L. (2004). Formação, manejo $e$ recuperação de pastagens em Rondônia. Porto Velho: Embrapa Rondônia. 215p.

Costa, N. L., Gianluppi, V., Braga, R. M. \& Bendahan, A. B. (2009). Alternativas tecnológicas para a pecuária de Roraima. Boa Vista: Embrapa Roraima. 35p. (Documentos, 19).

Costa, N. L., Moraes, A., Monteiro, A. L. G., Motta, A. C. V., Oliveira, R. A. \& Rodrigues, A. N. A. (2013). Forage productivity and morphogenesis of Axonopus aureus under different nitrogen fertilization rates. Revista Brasileira de Zootecnia, 42, 541-548.

Difante, G. S., Nascimento Júnior, D., Silva, S. C., Euclides, V. P. B. \& Montagner, D. B. (2011). Características morfogênicas e estruturais do capim-marandu submetido a combinações de alturas e intervalos de corte. Revista Brasileira de Zootecnia, 40, 955-963.

Lemaire, G., Hodgson, J. \& Chabbi, A. (2011). Grassland productivity and ecosystem services. Cabi, Wallingford.

Nabinger, C. \& Carvalho, P. F. C. (2009). Ecofisiología de sistemas pastoriles: aplicaciones para su sustentabilidad. Agrociencia, 13, 18-27.

Santos, M. E. R., Fonseca, D. M., Braz, T. G. S., Silva, S. P., Gomes, V. M. \& Silva, G. P. (2011). Características morfogênicas e estruturais de perfilhos de capim-braquiária em locais do pasto com alturas variáveis. Revista Brasileira de Zootecnia, 40, 535-542.

Santos, M. R., Fonseca, D. M., Gomes, V. M., Silva, S. P., Silva, G. P. \& Reis, M. (2012). Correlações entre características morfogênicas e estruturais em pastos de capim-braquiária. Ciência Animal Brasileira, 13, 49-56.

Sarmiento, G., Silva, M. P., Naranjo, M. E. \& Pinillos, M. (2006). Nitrogen and phosphorus 
as limiting factors for growth and primary production in a flooded savanna in the Venezuelan Llanos. Journal of Tropical Ecology, 22, 203-212.

Sousa, B. M. L., Nascimento Júnior, D., Rodrigues, C. S., Monteiro, H. C. F., Silva, S. C., Fonseca, D. M. \& Sbrissia, A. F. (2011). Morphogenetic and structural characteristics of Xaraes palisadegrass submitted to cutting heights. Revista Brasileira de Zootecnia, 40, 53-59.

Zanini, G. D., Santos, G. T. \& Sbrissia, A. F. (2012). Frequencies and intensities of defoliation in Aruana guineagrass swards: Morphogenetic and structural characteristics. Revista Brasileira de Zootecnia, 41, 18481857.
Recebido em Janeiro 4, 2016

Aceito em Fevereiro 2, 2016

License information: This is an open-access article distributed under the terms of the Creative Commons Attribution License, which permits unrestricted use, distribution, and reproduction in any medium, provided the original work is properly cited. 